\title{
Neuromodulation for Drug-Refractory Epilepsy and Chronic Heart Failure: Targets, Delivery, Composition and Titration
}

\author{
Inder S Anand, MD, FRCP, DPhil (Oxon) ${ }^{1 *}$, Marvin A Konstam, MD², Jeffrey L Ardell, PhD ${ }^{3}$, Imad Libbus, \\ $\mathrm{PhD}^{4}$, Lorenzo A DiCarlo, $\mathrm{MD}^{4}$ and Douglas L Mann, $M D^{5}$
}

\author{
${ }^{1}$ Division of Cardiology, University of Minnesota, Minneapolis, MN, USA \\ ${ }^{2}$ The Cardiovascular Center, Tufts Medical Center, Boston, MA, USA \\ ${ }^{3}$ Cardiac Arrhythmia Center, University of California, Los Angeles, CA, USA \\ ${ }^{4}$ LivaNova Inc., Houston, TX, USA
}

${ }^{5}$ Center for Cardiovascular Research, School of Medicine, Washington University, St. Louis, MO, USA

*Corresponding author: Inder S Anand, MD, FRCP, DPhil (Oxon), Division of Cardiology, VA Medical Center, University of Minnesota, Cardiology 111-C, One Veterans Drive, Minneapolis, MN 55417, USA, Tel: +1-(612)-467-3663, Fax: +1-(612)970-5899

\begin{abstract}
Background: Vagus nerve stimulation (VNS) is used currently in the management of drug-refractory epilepsy (DRE), and is in development for treating chronic heart failure (HF). $\mathrm{HF}$ is accompanied by autonomic nervous system (ANS) dysfunction, consisting of sustained sympathetic hyper-activation and withdrawal of parasympathetic tone, and associated with progressive worsening of cardiovascular (CV) function and increased morbidity and mortality. We sought to compare how VNS is utilized for DRE and may potentially be employed for HF.
\end{abstract}

Methods: A search was conducted in PubMed for all published articles using the search terms "vagus" OR "vagal" OR "VNS" AND "epilepsy", and a separate search used similar search terms AND "heart failure". Further filtering yielded the articles available as free full text, and clinical trial, prospective study, and retrospective study publications. The final selection of clinical trial/study publications in epilepsy or HF was based upon at least 3 months of follow-up and exclusion of pediatric studies. Additional information was obtained subsequently from references within these publications, and technical manuals available in the public domain.

Results: For DRE, VNS is administered via the left cervical vagus nerve (CVN), and directed toward the central nervous system (CNS). No known biomarkers exist currently for real-time VNS adjustment. VNS is titrated empirically, using large and infrequent adjustments of VNS intensity and based upon achieving long term reduction in seizure frequency (RSF) and/or reaching the maximum tolerated
VNS intensity. A significantly greater mean RSF has been observed after 3 months of VNS delivery using pulse frequency $30 \mathrm{~Hz}$ and duty cycle $9 \%$ (30 seconds on, 5 minutes off) when compared to using $1 \mathrm{~Hz}$ and duty cycle $14 \%$ (30 seconds on, 3 minutes off). A further reduction of mean RSF has been observed after 12 months by increasing the pulse frequency at 3 months from 1 to $30 \mathrm{~Hz}$.

For HF, VNS has been administered via the left or right $\mathrm{CVN}$, targeting both the CNS and/or peripheral hierarchical autonomic reflex arcs that control cardiovascular function. Real-time changes in heart rate dynamics (HR and $H R$ variability) have served as a biomarker of ANS engagement (ANSE) for titration. Titration to ANSE has been completed in 2.5 (median; range 1.6-3.6) months using 10 $\mathrm{Hz}$ and duty cycle $23 \%$ (18 seconds on, 1 minute off), using smaller and more frequent adjustments of VNS intensity, and has been associated with significant improvements after 6 months in HR, HR variability, CV function, and HF symptoms. Use of $1 \mathrm{~Hz}$ may have less clinical efficacy than occurs with a higher pulse frequency, however, intolerable side effects have occurred before ANSE using $20 \mathrm{~Hz}$.

Conclusions: Neuromodulation for the treatment of epilepsy and HF is not a one-size-fits-all therapy. The magnitude of long term reduction of seizure frequency in DRE, and improvements in function and symptoms in HF, may have a potential dose-dependent relationship to the intensity of VNS delivery. However, VNS for these disorders differs in neurologic targets, the technology platforms and paradigms used for titration, and the time and the frequency needed for up-titrating VNS.

Citation: Anand IS, AKonstam M, Ardell JL, Libbus I, DiCarlo LA, et al. (2019) Neuromodulation for Drug-Refractory Epilepsy and Chronic Heart Failure: Targets, Delivery, Composition and Titration. Int J Neurol Neurother 6:091. doi.org/10.23937/2378-3001/1410091

Accepted: September 21, 2019: Published: September 23, 2019

Copyright: (c) 2019 Anand IS, et al. This is an open-access article distributed under the terms of the Creative Commons Attribution License, which permits unrestricted use, distribution, and reproduction in any medium, provided the original author and source are credited. 


\section{Keywords}

Autonomic nervous system, Autonomic regulation therapy, Neuromodulation, Vagus nerve stimulation

\section{Abbreviations}

ANS: Autonomic Nervous System; ART: Autonomic Regulation Therapy; HF: Chronic Heart Failure; CNS: Central Nervous System; CV: Cardiovascular; CVN: Cervical Vagus Nerve; DRE: Drug-Refractory Epilepsy; GDMT: Guideline Directed Medical Therapy; HFrEF: Heart Failure with Reduced Left Ventricular Ejection Fraction; HR: Heart Rate; VNS: Vagus Nerve Stimulation

\section{Introduction}

Vagus nerve stimulation (VNS) for neuromodulation [1] is used currently for the management of patients with drug-refractory epilepsy [2,3], and is an investigational therapy being evaluated in patients with chronic heart failure (HF). HF is known to be accompanied by autonomic dysfunction that results in sympatho-vagal imbalance with sustained sympathetic hyperactivation and withdrawal of parasympathetic tone [4-6]. This results in progressive cardiac dysfunction that is manifested as worsening HF [7-10] with increased risk of mortality and morbidity $[11,12]$.

Although remarkable progress has been made to correct the sympatho-vagal imbalance in HF by blocking the deleterious effects of sympathetic hyperactivation with beta-blockers and andrenin-angiotensin-aldosterone system (RAAS) inhibitors with considerable success, $\mathrm{HF}$ remains a progressive, deteriorating condition and there are as yet no approved therapies to improve parasympathetic tone. To overcome this gap, VNS is in development as a possible therapeutic option.

For epilepsy, VNS is utilized to decrease the frequency of seizures [2]. In HF, early data shows that VNS potentially improves symptoms, cardiac function, and quality of life, and its effects on long-term outcomes including mortality and hospitalization for heart failure are being assessed [13]. In this article, we describe how VNS has been deployed for its intended objectives in the treatment of epilepsy and HF.

\section{Materials and Methods}

A search was conducted in PubMed for all published articles using the search terms "vagus" OR "vagal' OR "VNS" AND "epilepsy", and a separate search used similar search terms AND "heart failure". Further filtering yielded the articles available as free full text, and clinical trial, prospective study, and retrospective study publications. The final selection of clinical trial/study publications in epilepsy or HF was based upon at least 3 months of follow-up and ex-

Table 1: Clinical studies of VNS in patients with drug-refractory epilepsy.

\begin{tabular}{|c|c|c|c|c|c|c|c|c|c|c|c|}
\hline First Author/Year & Type & Scope & Design & Control & $\mathbf{N}$ & $\begin{array}{l}\text { PF } \\
(\mathrm{Hz})\end{array}$ & $\begin{array}{l}\text { PW } \\
\text { (psec) }\end{array}$ & $\begin{array}{l}\text { ON } \\
(\mathrm{sec})\end{array}$ & $\begin{array}{l}\text { OFF } \\
(\mathrm{min})\end{array}$ & $\begin{array}{l}\text { Curr } \\
(\mathrm{mA})\end{array}$ & $\begin{array}{l}\text { Titr } \\
\text { Freq } \\
\text { (wk) }\end{array}$ \\
\hline $\begin{array}{l}\text { Ben-Menachem } 1994 \\
\text { [14] }\end{array}$ & Pro & MC & Rand & Active & 67 & $20-50$ & 500 & $30-90$ & $5-10$ & $0.25-3.0$ & 4 \\
\hline George 1994 [15] & Pro & MC & Exten & None & 24 & $20-50$ & 500 & $30-90$ & $5-10$ & $0.25-3.0$ & \\
\hline $\begin{array}{l}\text { VNS Study Grp } 1995 \\
\text { [16] }\end{array}$ & Pro & MC & Rand & Active & 114 & $20-50$ & 500 & $30-90$ & $5-10$ & $0.25-3.0$ & 4 \\
\hline Salinski 1996 [17] & Pro & MC & Exten & None & 100 & $20-50$ & 500 & $30-90$ & $5-10$ & $0.25-3.0$ & \\
\hline Handforth 1998 [18] & Pro & MC & Rand & Active & 254 & 30 & 500 & 30 & 5 & $3.5(\max )$ & 4 \\
\hline Vonck 1999 [19] & Retro & MC & Observ & None & 15 & 30 & 500 & 30 & $5-10$ & $3(\max )$ & 4 \\
\hline DeGiorgio 2000 [20] & Pro & MC & Exten & None & 195 & $20-50$ & 500 & $30-90$ & $5-10$ & $0.25-3.0$ & \\
\hline Kawai 2002 [21] & Retro & SC & Observ & None & 15 & $20-127$ & $130-1000$ & $30-60$ & $5-10$ & $0.25-0.75$ & 12 \\
\hline Uthman 2004 [22] & Retro & SC & Observ & None & 25 & 30 & 750 & 30 & 3 & 1.75 (med) & 4 \\
\hline De Herdt 2007 [23] & Retro & MC & Observ & None & 138 & 30 & $250-500$ & 30 & $5-10$ & 1.8 (mean) & 4 \\
\hline Pakdaman 2011 [24] & Pro & SC & Observ & None & 44 & 30 & 250 & 30 & 3 & 1.5 & 4 \\
\hline Ghaemi 2010 [25] & Retro & SC & Observ & None & 144 & 30 & 500 & 30 & 5 & 3.5 (max) & 4 \\
\hline Ching 2013 [26] & Retro & SC & Observ & None & 100 & 25 & 307 & 28.9 & 4 & 2.0 (mean) & NR \\
\hline $\begin{array}{l}\text { Ben-Menachem } 1999 \\
\text { [27] }\end{array}$ & Pro & SC & Observ & None & 64 & NR & NR & NR & NR & NR & NR \\
\hline Amar 1999 [28] & Pro & MC & Registr & Active & 4743 & NR & NR & NR & NR & NR & NR \\
\hline Chavel 2003 [29] & Pro & SC & Observ & None & 23 & NR & NR & NR & NR & NR & NR \\
\hline Elliot 2011 [30] & Retro & SC & Observ & None & 65 & NR & NR & NR & NR & NR & NR \\
\hline
\end{tabular}

Note: Curr: Current; Exten: Extension; Freq: Frequency; Min: Minutes; Grp: Group; Hz: Hertz; mA: Milliamperes; MC: Multicenter; Max: Maximum; Med: Median; N: Number; NR: Not Reported; Observ: Observational; PF: Pulse Frequency; Pro: Prospective; PW: Pulse Width; Rand: Randomized; Registr: Registry; Retro: Retrospective; SC: Single Center; Sec: Seconds; Titr Freq: Titration Frequency; $\mu$ sec: Microseconds; wk: Weeks. 
clusion of pediatric studies and redundancies. Additional information was obtained subsequently from references within these publications, and technical manuals available in the public domain.

\section{Results}

The consort diagrams in Figure 1 provide the search results, and Table 1 and Table 2 provide an overview of the clinical studies that were identified for DRE and HF, respectively.

\section{VNS delivery}

VNS is delivered utilizing a pulse generator, an electrode lead that surrounds the cervical vagus nerve (CVN), and an external programming system used to change the generator settings for stimulating the vagus nerve. The electrode lead requires no intraoperative mapping for placement. The generator and lead deliver electrical stimulation to axons that comprise the CVN; this includes approximately $80 \%$ afferent and $20 \%$ parasympathetic preganglionic efferent projections [37-39].

VNS parameters include intensity of the current stimulus, its polarity, and mode of delivery. VNS intensity comprises the combination of the pulse amplitude, pulse frequency, pulse duration (width), and duty cycle. The duty cycle consists of a period of VNS ("on-time") alternating with a period of no stimulation ("off-time"), is measured by dividing the duration of the on-time by the total cycle duration (i.e., on-time plus off-time) and is repetitive over time. The mode of delivery may
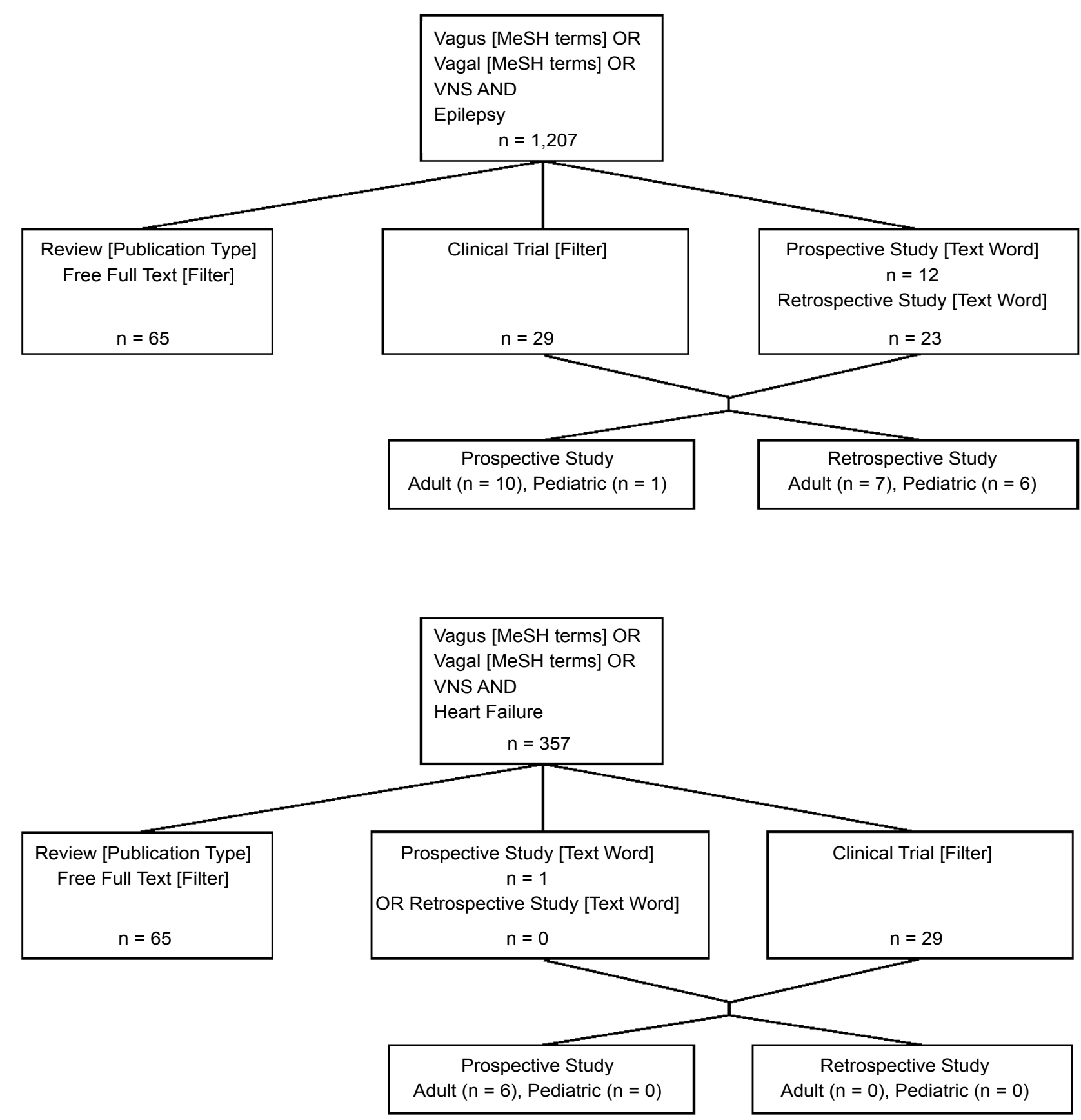

Figure 1: CONSORT diagrams of search results for DRE and HF. 
Table 2: Clinical studies of VNS in patients with heart failure and reduced ejection fraction.

\begin{tabular}{|c|c|c|c|c|c|c|c|c|c|c|c|}
\hline First Author/Year & Type & Scope & Design & Control & $\mathbf{N}$ & $\begin{array}{l}\text { PF } \\
(\mathrm{Hz})\end{array}$ & $\begin{array}{l}\text { PW } \\
\text { (psec) }\end{array}$ & $\begin{array}{l}\text { ON } \\
\text { (sec) }\end{array}$ & $\begin{array}{l}\text { OFF } \\
\text { (min) }\end{array}$ & $\begin{array}{l}\text { Curr } \\
\text { (mA) }\end{array}$ & $\begin{array}{l}\text { Titr } \\
\text { Freq } \\
\text { (wk) }\end{array}$ \\
\hline De Ferrari 2011 [31] & Pro & MC & Obs & None & 32 & $1-2$ & NR & Variable & Variable & $1.1-5.5(\max )$ & 1 \\
\hline $\begin{array}{l}\text { Premchand } 2014 \\
\text { [32] }\end{array}$ & Pro & MC & Rand & Active & 60 & 10 & 250 & 18 & 1.1 & $1.5-3.0$ (max) & $1-2$ \\
\hline Zanad 2014 [33] & Pro & MC & Rand & Sham & 96 & 20 & 300 & 10 & 0.8 & $\begin{array}{l}1.4 \text { (mean) }, 4.0 \\
(\max )\end{array}$ & 1 \\
\hline $\begin{array}{l}\text { Premchand } 2015 \\
\text { [34] }\end{array}$ & Pro & MC & Extens & Active & 49 & $\begin{array}{l}5 \\
10\end{array}$ & 250 & 18 & 1.1 & $1.5-3$ (max) & $1-2$ \\
\hline De Ferrari 2015 [35] & Pro & MC & Extens & Sham & 96 & 20 & 300 & 10 & 0.8 & $\begin{array}{l}1.4 \text { (mean), } 4.0 \\
(\max )\end{array}$ & 1 \\
\hline Gold 2016 [36] & Pro & MC & Rand & SOC & 707 & $1-2$ & NR & Variable & Variable & $1.1-5.5$ (max) & 1 \\
\hline
\end{tabular}

Note: SOC: Standard of Care (Guideline Directed Medical Therapy); All other abbreviations are the same as in Table 1.

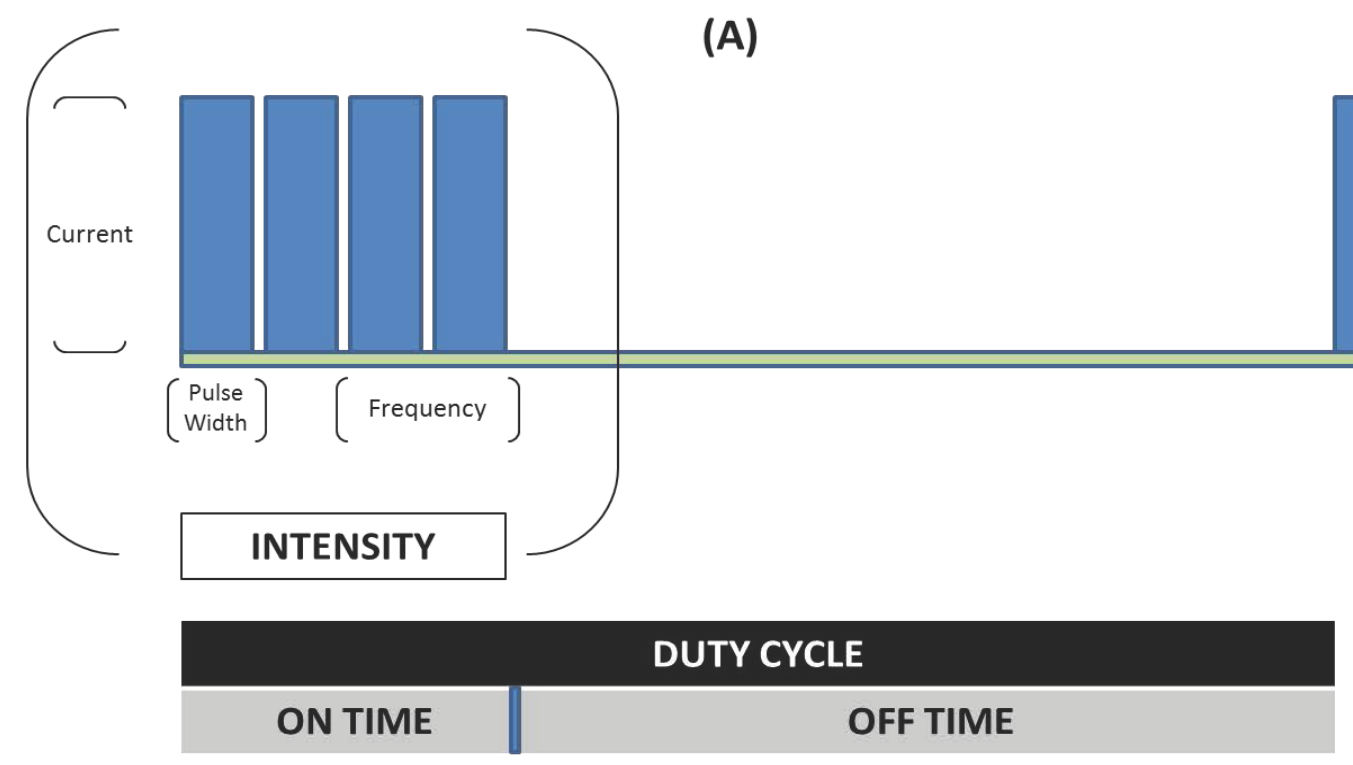

\section{(B)}

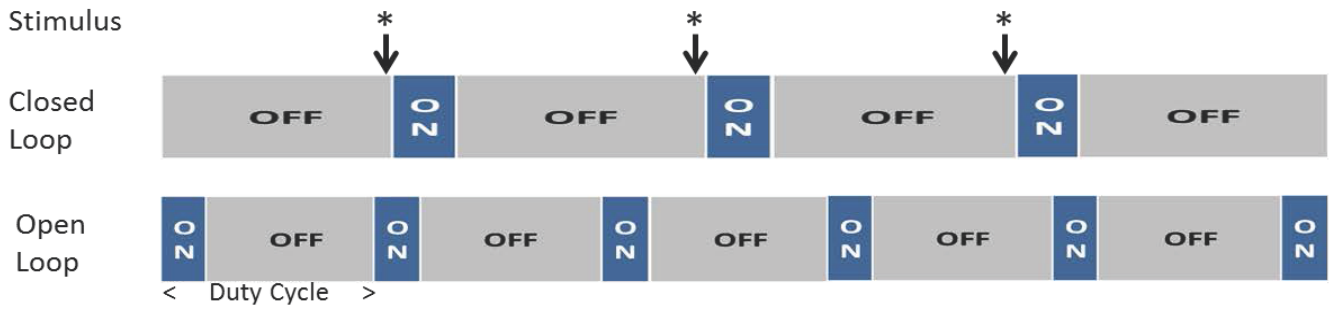

Figure 2: VNS delivery includes its intensity (a combination of pulse amplitude, pulse frequency, and pulse duration) and duty cycle (Panel A). The mode of delivery may be open-loop or closed loop (Panel B).

be closed-loop or open-loop. Closed-loop delivery comprises VNS that occurs only in response to an external stimulus. Open-loop delivery comprises VNS that occurs without any dependence on detection of an external stimulus (Figure 2).

\section{VNS for DRE}

For epilepsy, VNS is administered using open-loop delivery to the left cervical vagus nerve, and directed preferentially toward the central nervous system (CNS) by orienting the cathodic electrode toward the brain. There are as yet no known biomarkers that have been established for real-time titration of VNS for DRE into a therapeutic range. For this reason, a change in seizure frequency is the metric of choice to guide VNS titration [1-3].

The reduction of long term seizure frequency appears to be associated with the VNS intensity that is utilized. A significantly greater reduction in seizure fre- 
quency after 3 months has been observed with VNS delivery at "high" intensity when compared to VNS delivered at a "low" intensity ("high": Pulse frequency $30 \mathrm{~Hz}$; duty cycle 9\% [30 seconds on, 5 minutes off]; current 0.25-2.5 mA; "low": Pulse frequency $1 \mathrm{~Hz}$; duty cycle $14 \%$ [30 seconds on, 3 minutes off]; current 0.25 $2.5 \mathrm{~mA}$ ) $[16,18]$. Adjustment of VNS delivery to "high" intensity after 3 months at "low" intensity has been associated at 12 months with a significant subsequent reduction in mean seizure frequency [20], and a significant reduction in median seizure frequency has been reported when "high" intensity includes a duty cycle $>22 \%$ or a duty cycle with an off time of 1.1 minutes [40].

A clinical paradigm for VNS titration has evolved from these observations. Initially, pulse widths of 250 or 500 microseconds, and a pulse frequency of $30 \mathrm{~Hz}$ signal frequency are typically utilized while the output current is increased in $0.25 \mathrm{~mA}$ increments over the first
12 weeks of VNS Therapy. During this time, the duty cycle is $10 \%$ (i.e., 30 seconds on, 5 minutes off). After the device output current reaches 1.5 to $1.75 \mathrm{~mA}$, the duty cycle off-time is reduced, and seizure activity is monitored for an additional three months before additional adjustments are made $[41,42]$.

\section{VNS for HF}

Several alternative approaches have been explored for delivering VNS for the treatment of HF. VNS has been administered via the left or right CVN, and the technology platforms and methods for HF have differed from that for epilepsy (Figure 3A) [31$33,36,43-45]$. VNS polarity and software are configured to direct stimulation toward both the CNS and the periphery. The goal of VNS titration has been to objectively confirm autonomic engagement for cardiac control using sinoatrial node behavior as a biomarker. Changes in heart rate and heart rate variability have been targeted to confirm adequate
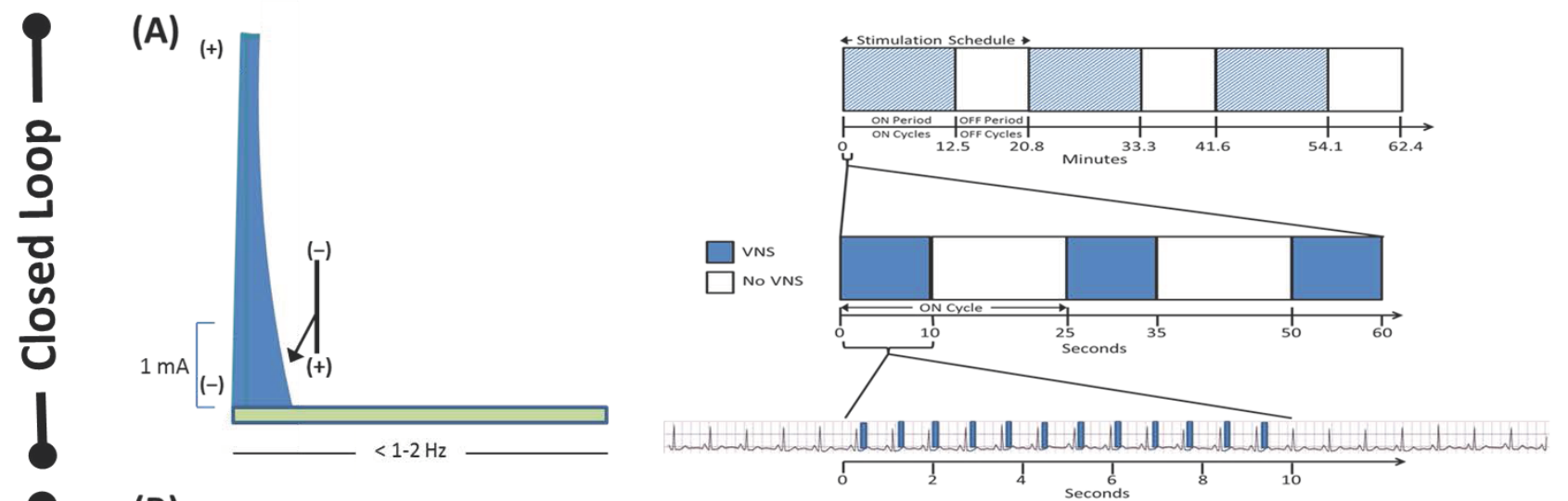

(B)
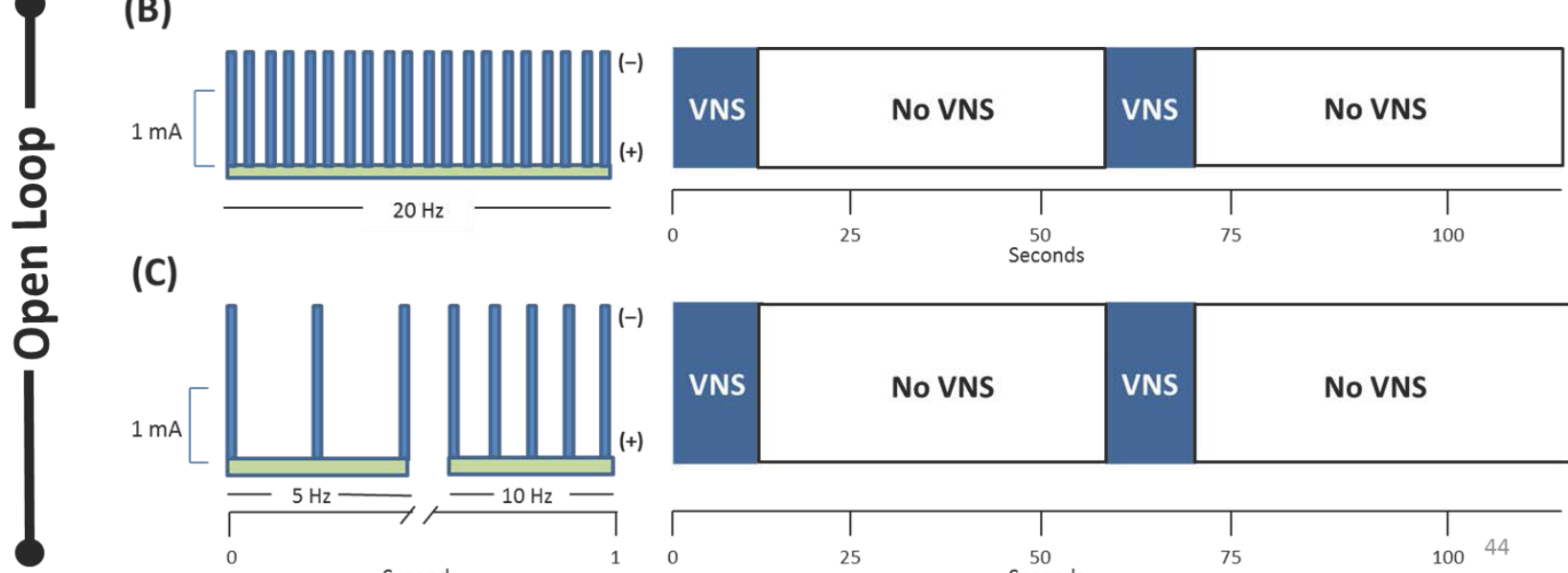

(C)
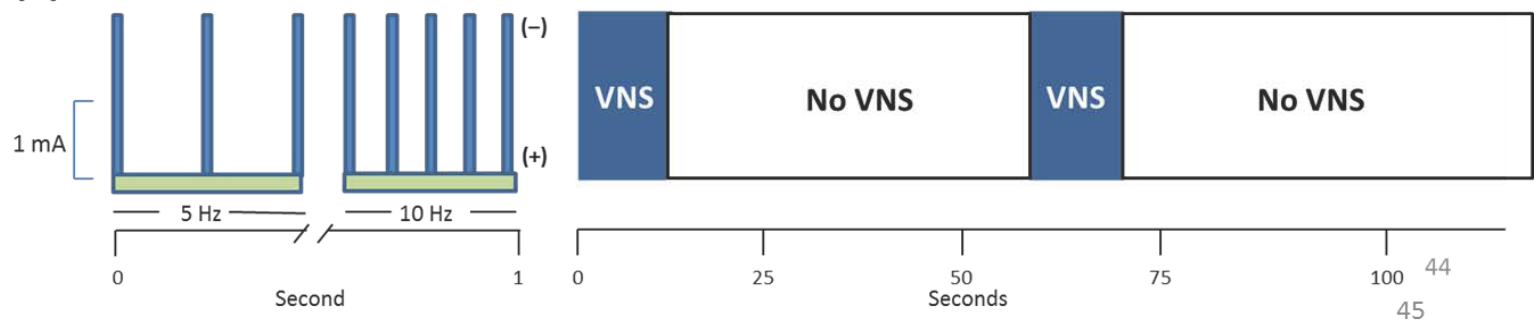

Figure 3A: Comparison of pulse frequencies, polarities, and duty cycles used for VNS delivery for the treatment of heart failure in INOVATE-HF (Panel A) [36] , NECTAR-HF (Panel B) [33] , and ANTHEM-HF (Panel C) [32,34]. The mode of VNS delivery was closed-loop in INOVATE-HF, and open-loop in the other studies. INOVATE-HF utilized a repetitive stimulation schedule for VNS delivery consisting of ON Cycles followed by OFF Cycles. Each cycle comprised an ON Time followed by an OFF Time. ON Cycles delivered VNS for detected R-waves during the ON Time and no VNS during the OFF Time. OFF Cycles delivered no VNS during the ON Time or the OFF Time. As an example, nominal stimulation schedule could be programmed to have a sequence of 30 ON Cycles followed by 20 OFF Cycles, a total duration of 25 seconds for each cycle, and delivery of a single VNS pulse for each detected R-wave during the first 10 seconds of each ON Cycle [38]. Administration of a single VNS pulse following at most $25 \%$ of detected R-waves would yield VNS delivery with a pulse frequency of $<1$ when administered during heart rates of 56-130 beats per minute. Administration of three VNS pulses as the maximum number of pulses allowable would yield a pulse frequency of $<2 \mathrm{~Hz}$. 
autonomic engagement [46]. Smaller and more frequent adjustments of VNS are employed to achieve autonomic engagement using a minimum effective VNS intensity, which allows titration to be completed in a shorter period of time (Table 2).

Some studies have evaluated VNS for HF utilizing open-loop stimulation in both afferent (toward the brain) and efferent direction (toward peripheral hierarchical autonomic reflex arcs) to control cardiovascular function [32,33]. Use of a pulse frequency of $20 \mathrm{~Hz}$ (Figure $3 A$, Panel $B$ ) has been reported to cause significant off-target effects that have made it intolerable to up titrate the stimulation current sufficiently to engage the ANS acutely, to achieve any significant long term changes in heart rate (HR) or parameters of $H R$ variability that would be indicative of autonomic engagement, or to attain sufficient subsequent improvement in signs and symptoms of HF $[33,35]$.

An alternative approach has used closed-loop stimulation that requires implantation of a right ventricular (RV) pacemaker lead for synchronizing VNS delivery with RV depolarizations [31,36,44]. This technology delivers VNS using a quasi-trapezoidal, charge balanced, asymmetric current pulse. An initial high-amplitude anodal phase is employed in an attempt to produce electrophysiological conduction block in the afferent direction. This is followed by a cathodic efferent phase with a current that is limited to an amplitude that is $5 \%$ of the amplitude of the initial phase [47]. VNS occurs intermittently, based on a repetitive and complex stimulation schedule (Figure $3 \mathrm{~A}$, Panel A) that administers VNS following at most $25 \%$ of detected R-waves [31,38]. This approach, coupled with the nominal delivery of a single pulse and no more than three pulses per sensed R-Wave for heart rates in the range of 56-130 beats/minute, administers VNS with a pulse frequency of less than 1-2 Hertz. To date, no long term changes in heart rate, consistent with modulation of the autonomic nervous system, has been observed with this technique [36].

In a third approach, VNS of the left and right cer- vical vagus have been compared in patients with HF and reduced left ventricular ejection fraction (HFrEF) [32]. The pulse frequencies for VNS in this study and its long term follow-up were selected to be similar to the spontaneous frequency observed in the efferent fibers of the vagal trunk (Figure 3A, Panel C) [48]. In this study, VNS successfully engaged the ANS in all patients while they were receiving standard of care treatment with Guideline Directed Medical Therapy (GDMT), as indicated by real-time changes in HR $[49,50]$. There was also a significant long term decrease in 24-hour heart rate and a significant increase in 24-hour heart rate variability, which are compatible with a sustained improvement in autonomic tone. VNS delivered in this manner was associated with significant increases when compared to baseline in left ventricular ejection fraction, 6-minute walk distance, NYHA classification, and quality of life [32]. A posthoc analysis has suggested that the greater magnitudes of clinical improvement in this study could be related to the differences in the technology platform and method that were used for VNS delivery when compared to the other two approaches (Figure 3B) [51]. A control arm of GDMT alone was not included in the ANTHEM-HF Pilot Study, however, therefore the overall effects that were observed may not have been solely attributable to ART alone. It is possible that at least some of the clinical improvements may have been related to the open label nature of the study and/or a Hawthorne effect that may have affected the more subjective assessments. Nevertheless, the overall direction of changes that have been observed in patient symptoms and function after $6,12,24$, and 42 months have been encouraging [32,34,52]. Confirmation of these findings are being investigated further in a larger, multi-center, randomized, placebo controlled study [13].

Figure 4 illustrates the methods used to deliver VNS in patients with epilepsy and patients investigated in the ANTHEM-HF Pilot Study. VNS for HF utilized a relatively larger duty cycle, a lower pulse frequency, and an output current that on average was higher than that used for epilepsy.

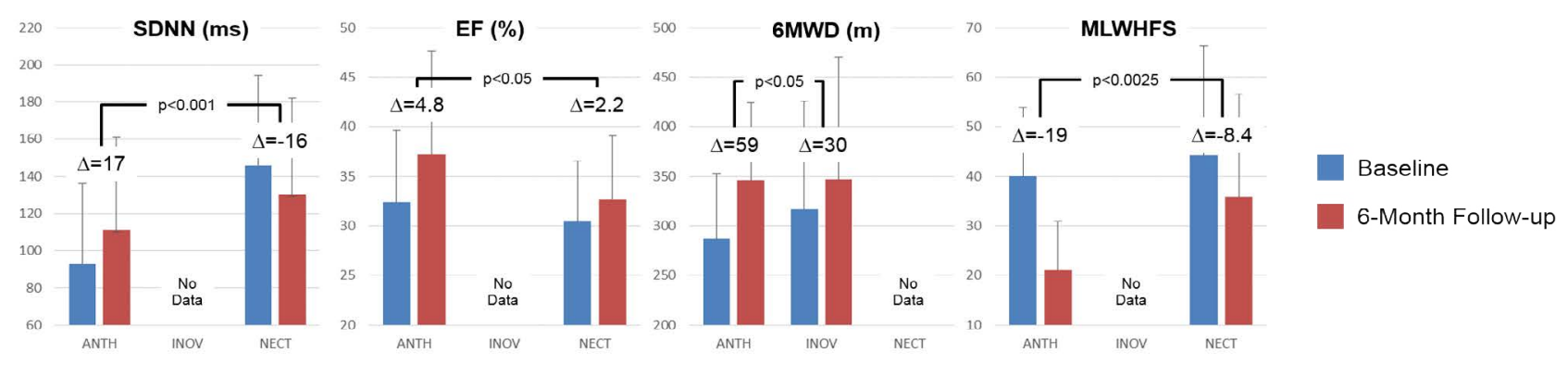

Figure 3B: Symptomatic and functional responses to VNS in INOVATE-HF, NECTAR-HF, and ANTHEM-HF. During chronic VNS in patients with HFrEF, heart rate and heart rate variability (SDNN) improved only in ANTHEM-HF. Significantly greater responses to VNS were observed in cardiac function \& HF symptoms in ANTHEM-HF when compared to INOVATE-HF and NECTAR-HF. 
(A)

\section{Epilepsy}

Maximum $2.5 \mathrm{~mA}$
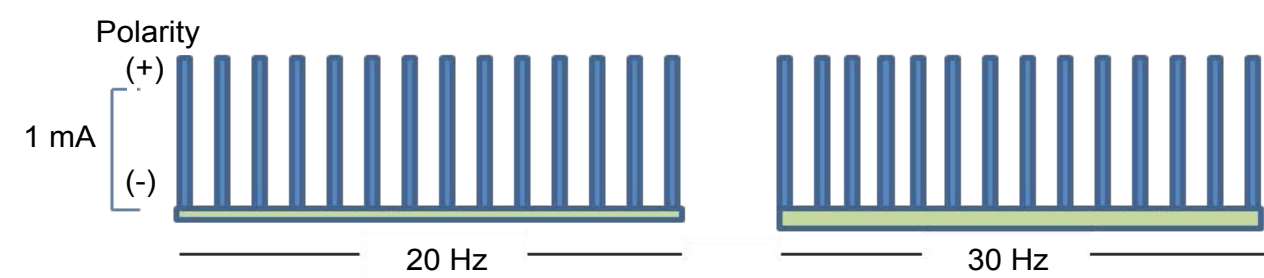

Median $1.25 \mathrm{~mA}$

\section{Heart Failure}

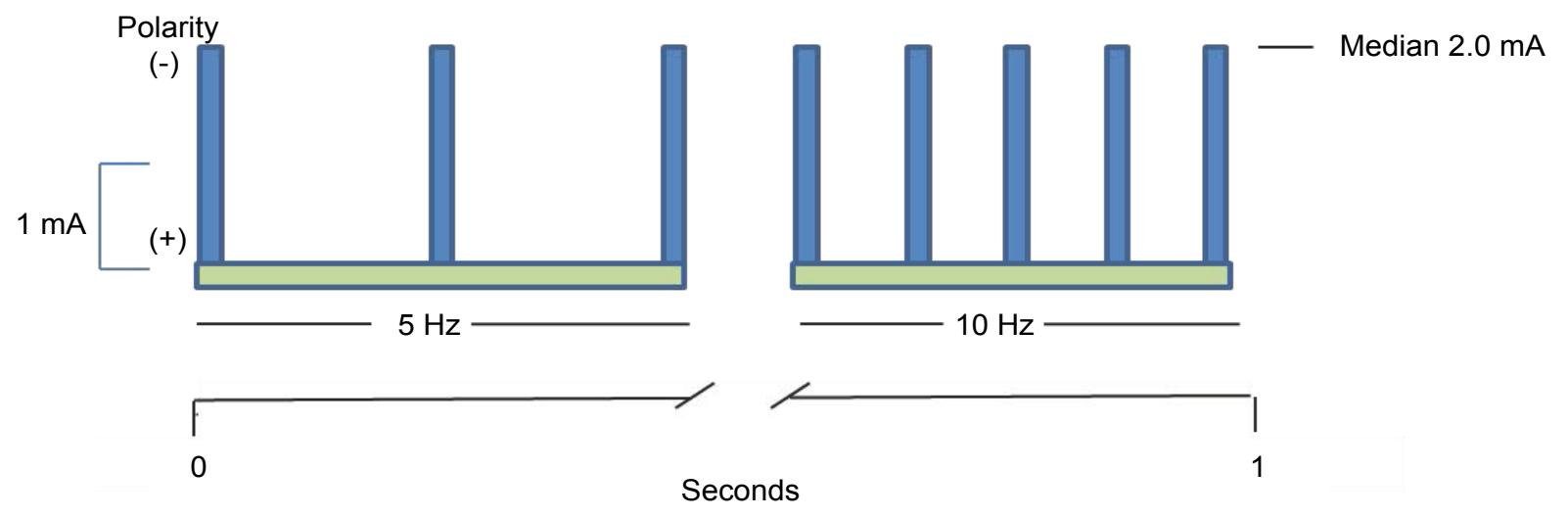

Figure 4A: Comparison of pulse frequencies, polarities, and amplitudes for VNS delivery in the treatment of epilepsy and as utilized in ANTHEM-HF.

\section{Discussion}

The hallmark of epilepsy is recurrent seizures, and it is the third most common neurological disorder in the world. Medications are inadequate for seizure control in up to $40 \%$ of afflicted patients [53]. Surgery may reduce or eliminate seizures in as many as $60 \%$ of patients with drug-refractory epilepsy. However, the remainder of patients continue to experience seizures that adversely impact their ability to conduct usual activities of daily living $[54,55]$. Long-term consequences can include increased seizure severity [56], seizure-related injuries [57], increased healthcare utilization [58], and increased mortality.

The hallmarks of HF include progressive deterioration of cardiac function, worsening symptoms that ultimately impair the ability to conduct activities of daily living, and a reduced life-span. Despite the remarkable progress that has been made in the use of pharmaco- logic therapy and devices for $\mathrm{HF}$, the overall prognosis of patients with $\mathrm{HF}$ still remains poor. In many patients, cardiac dysfunction and clinical manifestations of heart failure persist and worsen in conjunction with chronic dysregulation of the ANS [10].

In the more established domain of VNS for the treatment of epilepsy, the clinical benefits of VNS have been associated with interaction with the CNS [1]. The locus coeruleus and the dorsal raphe nucleus appear to play prominent roles in putative effects. Both of these have widespread projections to the brain and spinal cord, and release neuromodulators with robust antiepileptic effects during acute and chronic VNS. VNS-induced seizure suppression is abolished when they are interrupted [59].

VNS for HF is based on the hypothesis that down-regulation of the parasympathetic nervous system contributes to the progression of HF. VNS may 
(B)
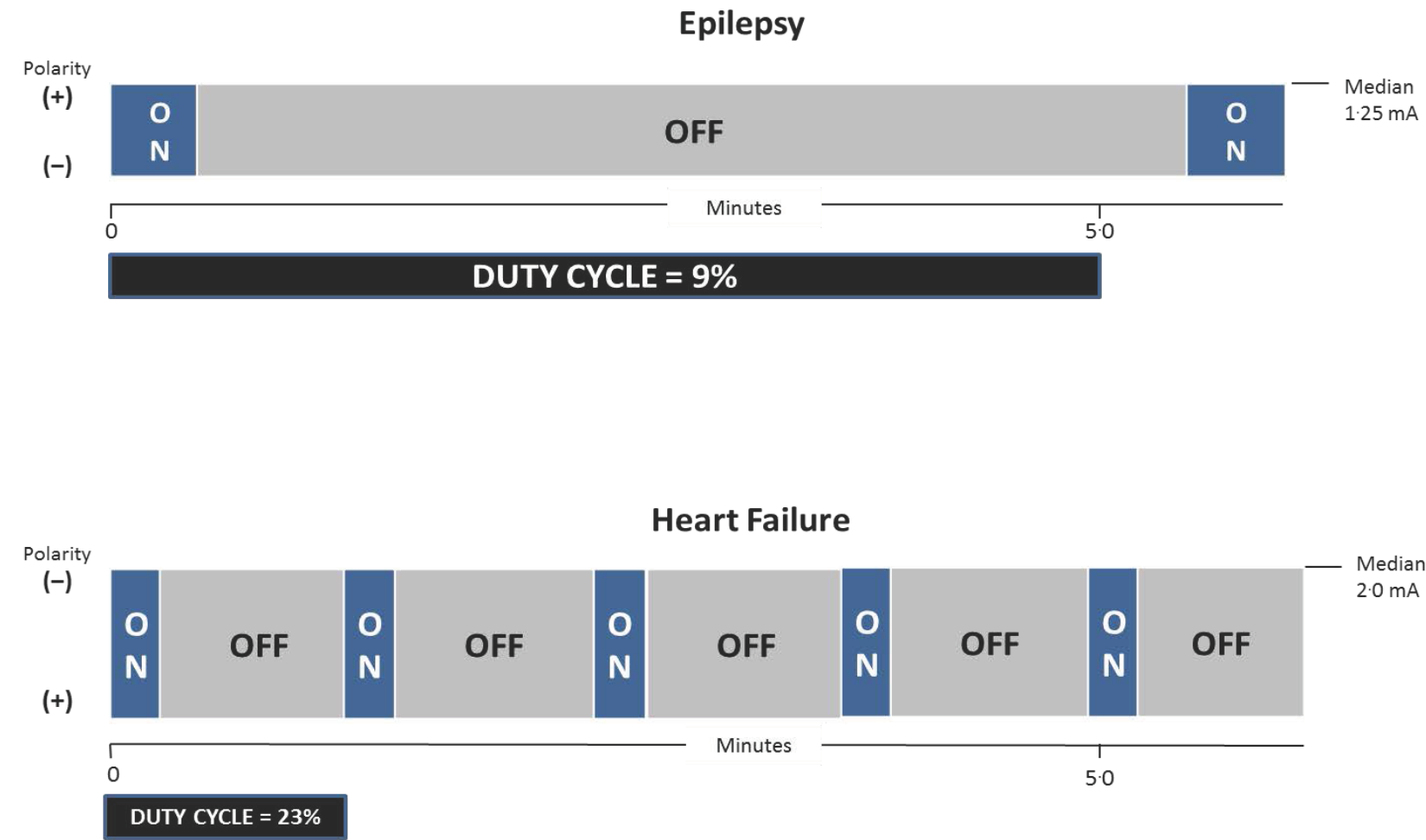

Figure 4B: Comparison of duty cycles for VNS delivery in the treatment of epilepsy $[41,42]$ and as utilized in ANTHEM-HF and its long-term followup [32,34,52].

benefit patients with HF by increasing parasympathetic activity, and a different paradigm is required for VNS administration and titration to achieve these objectives. Experimental studies have confirmed that VNS exerts anti-adrenergic effects by pre- and post-junctional interactions at end-terminus projections onto cardiomyocytes, and restoring synaptic efficacy within the intrinsic cardiac nervous system [60-63]. The objective of VNS for HF is the engagement of neurologic targets that include the CNS and ANS at multiple levels (Figure 5) [64-67]. Reaching a critical level of vagal stimulation appears to be essential in promoting beneficial modulation of central and peripheral autonomic nervous system (ANS) activity [46]. In order to achieve the appropriate level of autonomic engagement in a timely manner with the minimum effective VNS intensity, frequent programming and gradual adjustments of VNS pulse amplitude, pulse width, and pulse frequency (referred to as "titration") are required. VNS can be titrated to produce an immediate, relatively small change in beat-to-beat distribution of R-R intervals during the ON time of the VNS duty cycle. This change has been observed to correlate with the current amplitude of VNS. During titration of right CVNS, a strong correlation has been observed between stimulus intensity and a Poincaré map parameter of standard deviation, which is associated with high-frequency heart rate variability. The effect of VNS on instantaneous heart rate has consisted of a shift in the centroid of the beat-to-beat cloud distribution of $\mathrm{R}-\mathrm{R}$ intervals (Figure 6), with a tight coupling in beat-to-beat dynamics during VNS stimulation that has been directly related to stimulation intensity. This has provided a useful measurement for confirming an appropriate level of autonomic engagement [50]. This coupling has been achieved and maintained without any long-term side effects $[50,68]$, and has been associated with significant and clinically meaningful improvements in $\mathrm{HF}$ symptoms, exercise capacity, and cardiac function [32,34,52].

Past and current development of implantable and non-implantable VNS has also occurred for other indications such as major depression, anxiety, arrhythmias, cluster headache, cognition and brain injury, inflammation and autoimmune diseases, ischemia and myocardial infarction, migraine, obesity, pain, and rheumatoid arthritis $[69,70]$. This report was not intended to be an exhaustive review of all of these conditions, other conditions that might be amenable to neuromodulation, or alternative methods that might be used for modulating the nervous system. The objective of this report was to describe VNS as it is currently approved for use in the management of 


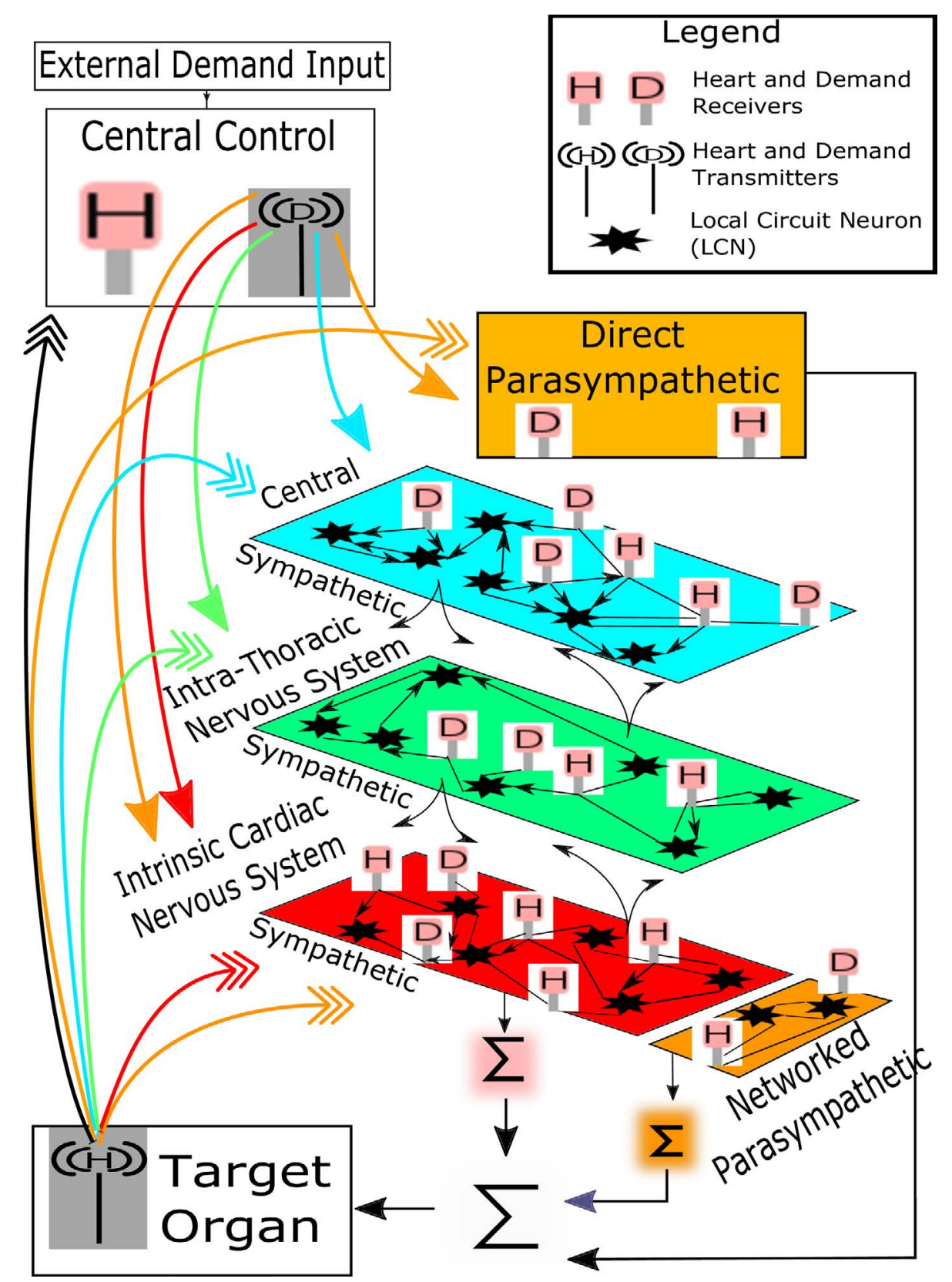

Figure 5: Cardiac function and homeostasis includes processes within the central nervous system as well as local circuit neurons for networked control within the peripheral nervous system. Network interactions occur within the local circuit neural (LCN) populations. These integrate activities within and between peripheral ganglia and the central nervous system subserve reflex control of the heart. The intrinsic cardiac nervous system possesses sympathetic (Sympath) and parasympathetic (Parasym) efferent post-ganglionic neurons, local circuit neurons, (LCN) and afferent (Aff.) neurons. The intrathoracic extracardiac nervous system is comprised of ganglia containing afferent neurons, LCN, and sympathetic efferent post-ganglionic neurons. Cardiovascular heart rate and demand inputs are conveyed centrally via dorsal root (DRG), nodose, and petrosal ganglia subserving spinal cord (C-cervical, T-thoracic), brainstem, and higher center reflexes for hemostatic maintenance. From Kember G. PLosONE 2017 (with permission) [65].

DRE, and to compare this with the more recent use of VNS in patients with HFrEF, in order to illustrate how VNS for these disorders differs in neurologic targets, the technology platforms and paradigms used for titration, and the time and the frequency needed for up-titrating VNS to achieve its intended therapeutic effects.

\section{Conclusions}

Neuromodulation for the treatment of epilepsy and $\mathrm{HF}$ is not a one-size-fits-all therapy. The magnitude of long term reduction of seizure frequency in
DRE, and improvements in function and symptoms in $\mathrm{HF}$, seem to have a potential dose-dependent relationship to the intensity of VNS that is utilized. However, the delivery of VNS for these disorders differs in neurologic targets, the technology platforms and paradigms used for titration, and the time and the frequency needed for up-titrating VNS.

Because no biomarker currently exists to guide VNS titration for DRE, VNS titration is based upon observing a reduction in seizure frequency or reaching the maximum tolerated VNS intensity. Increasing 


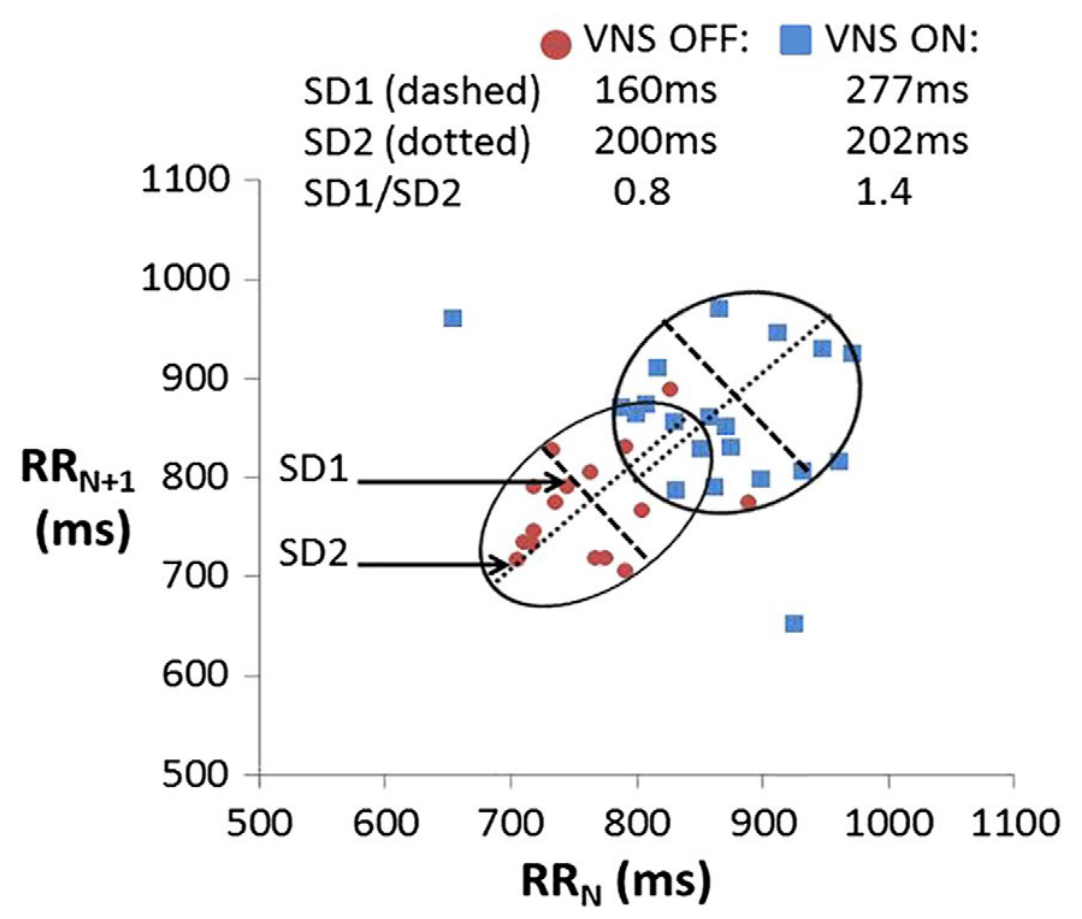

Figure 6: Quantitative beat-to-beat (RR) analysis of heart rate dynamics using the Poincaré method. There is a close coupling between the "ON" periods of VNS stimulation in humans with the beat-to-beat dynamics. During the right-sided VNS "ON" time, there is an upward shift and expansion of the beat-distribution cloud, reflected in an increase in SD1, and an increase in the average R-R intervals, consistent with an increase in parasympathetic input. Abbreviations: $m_{s}$ : Milliseconds; RR The time between two successive R-waves (ventricular electrograms); $R_{\mathrm{N}_{+1}}$ : The time between the next two successive R-waves; SD1: Instantaneous beat-to-beat variability in successive RR intervals; SD2: Standard deviation of continuous beat-to-beat R-R interval variability; SD1/SD2: A measure of autonomic balance with higher values correlating with vagal tone preponderance. From Libbus I. J Electrocardiol 2017 (with permission) [50].

data suggest that changes in heart rate dynamics may serve as a biomarker for confirming satisfactory ANS engagement during VNS up-titration to ameliorate HF. The minimum effective VNS intensity that achieves this engagement may be sufficient, and the completion of up-titration is possible in a shorter period of time if smaller and more frequent adjustments of VNS intensity are used. Strong and favorable clinical responses were observed with the approach used in the ANTHEM-HF Pilot Study. A similar paradigm is being tested in an international, multicenter, randomized controlled study of right CVNS to evaluate the role of ART in patients with HFrEF.

\section{Acknowledgements}

No funding was provided and no medical writer was used for the preparation of this manuscript. I. Anand and M. Konstam are contracted to LivaNova as cardiovascular consultants, and J. Ardell is contracted to LivaNova as a neurocardiology consultant. I. Libbus and L. DiCarlo are employees and shareholders of LivaNova USA Incorporated. M. Konstam is the Chair, and I. Anand and D. Mann are members, of the Steering Committee for the ANTHEM-HFrEF Pivotal Study.

\section{Authors' Contribution}

All authors contributed equally to the writing of this manuscript, had full access to all of the sources for this article, and shared final responsibility for the decision to submit it for publication.

\section{Sources of Support}

None.

\section{References}

1. Johnson RL, Wilson CG (2018) A review of vagus nerve stimulation as a therapeutic intervention. J Inflamm Res 11: 203-213.

2. Ben-Menachim E (2002) Vagus-nerve stimulation for the treatment of epilepsy. Lancet Neurol 1: 477-482.

3. Van Ness PC (2002) Therapy for the epilepsies. Arch Neurol 59: $732-735$.

4. Zhang DY, Anderson AS (2014) The sympathetic nervous system and heart failure. Cardiol Clin 32: 33-45.

5. Eckberg DL, Drabinsky M, Braunwald E (1971) Defective cardiac parasympathetic control in patients with heart failure. N Engl J Med 285: 877-883.

6. Brunner-La Rocca HP, Esler MD, Jennings GL, Kaye DM (2001) Effects of cardiac sympathetic nervous activity on mode of death in congestive heart failure. Eur Heart $\mathrm{J} 22$ : 1136-1143.

7. Loffelholz K, Pappano AJ (1985) The parasympathetic neuroeffector junction of the heart. Pharmacol Rev 37: 1-24.

8. Lara A, Damasceno DD, Pires R, Gros R, Gomes ER, et al. (2010) Dysautonomia due to reduced cholinergic neurotransmission causes cardiac remodeling and heart failure. Mol Cell Biol 30: 1746-1756. 
9. Kanazawa H, leda M, Kimura K, Arai T, Kawaguchi-Manabe $\mathrm{H}$, et al. (2010) Heart failure causes cholinergic transdifferentiation of cardiac sympathetic nerves via gp130-signaling cytokines in rodents. J Clin Invest 120: 408-421.

10. Joffe SW, Webster K, McManus DD, Kiernan MS, Lessard $D$, et al. (2013) Improved survival after heart failure: A community-based perspective. J Am Heart Assoc 2: e000053.

11. Nolan J, Batin PD, Andrews R, Lindsay SJ, Brooksby P, et al. (1998) Prospective study of heart rate variability and mortality in chronic heart failure: Results of the United Kingdom heart failure evaluation and assessment of risk trial (UK-heart). Circulation 98: 1510-1516.

12. La Rovere MT, Bigger JT, Marcus FI, Mortara A, Schwartz PJ (1988) Baroreflex sensitivity and heart-rate variability in prediction of total cardiac mortality after myocardial infarction. ATRAMI (Autonomic Tone and Reflexes After Myocardial Infarction) Investigators. Lancet 351: 478-484.

13. ANTHEM-HFrEF Pivotal Study (NCT03425422).

14. Ben-Menachem E, Manon-Espaillat R, Ristanovic R, Wilder BJ, Stefan H, et al. (1994) Vagus nerve stimulation for treatment of partial seizures: 1. A controlled study of effect on seizures. First International Vagus Nerve Stimulation Study Group. Epilepsia 35: 616-626.

15. George R, Salinsky M, Kuzniecky R, Rosenfeld W, Bergen D, et al. (1994) Vagus nerve stimulation for treatment of partial seizures: 3 . Long-term follow-up on first 67 patients exiting a controlled study. First International Vagus Nerve Stimulation Study Group. Epilepsia 35: 637-643.

16. (1995) A randomized controlled trial of chronic vagus nerve stimulation for treatment of medically intractable seizures. The Vagus Nerve Stimulation Study Group. Neurology 45: 224-230.

17. Salinsky MC, Uthman BM, Ristanovic RK, Wernicke JF, Tarver WB (1996) Vagus nerve stimulation for the treatment of medically intractable seizures. Results of a 1-year open-extension trial. Vagus Nerve Stimulation Study Group. Arch Neurol 53: 1176-1180.

18. Handforth A, DeGiorgio CM, Schachter SC, Uthman BM, Naritoku DK, et al. (1998) Vagus nerve stimulation therapy for partial-onset seizures: A randomized active-control trial. Neurology 51: 48-55.

19. Vonck K, Boon P, D'Havé M, Vandekerckhove T, O'Connor $S$, et al. (1999) Long-term results of vagus nerve stimulation in refractory epilepsy. Seizure 8: 328-334.

20. DeGiorgio CM, Schachter SC, Handforth A, Salinsky M, Thompson J, et al. (2000) Prospective long-term study of vagus nerve stimulation for the treatment of refractory seizures. Epilepsia 41: 1195-1200.

21. Kawai K, Shimizu H, Maehara T, Murakami H (2002) Outcome of long-term vagus nerve stimulation for intractable epilepsy. Neurol Med Chir (Tokyo) 42: 481-489.

22. Uthman BM, Reichl AM, Dean JC, Eisenschenk S, Gilmore $R$, et al. (2004) Effectiveness of vagus nerve stimulation in epilepsy patients: A 12-year observation. Neurology 63: 1124-1126.

23. De Herdt V, Boon P, Ceulemans B, Hauman H, Lagae L, et al. (2007) Vagus nerve stimulation for refractory epilepsy: A Belgian multicenter study. Eur J Paediatr Neurol 11: 261-269.

24. Pakdaman $\mathrm{H}$, Amini Harandi A, Abbasi M, Karimi M, Arami MA, et al. (2016) Vagus nerve stimulation in drug resistant epilepsy: The efficacy and adverse effects in a 5-year fol- low-up study in Iran. Neurol Sci 37: 1773-1778.

25. Ghaemi K, Elsharkawy AE, Schulz R, Hoppe M, Polster T, et al. (2010) Vagus nerve stimulation: Outcome and predictors of seizure freedom in long-term follow-up. Seizure 19: 264-268.

26. Ching J, Khan S, White P, Reed J, Ramnarine D, et al. (2013) Long-term effectiveness and tolerability of vagal nerve stimulation in adults with intractable epilepsy: A retrospective analysis of 100 patients. $\mathrm{Br} \mathrm{J}$ Neurosurg 27: 228-234.

27. Ben-Menachem E, Hellström K, Waldton C, Augustinsson LE (1999) Evaluation of refractory epilepsy treated with vagus nerve stimulation for up to 5 years. Neurology 52: 1265-1267.

28. Amar AP, DeGiorgio CM, Tarver WB, Apuzzo ML (1999) Long-term multicenter experience with vagus nerve stimulation for intractable partial seizures: Results of the XE5 trial. Stereotact Funct Neurosurg 73: 104-108.

29. Chavel SM, Westerveld M, Spencer S (2003) Long-term outcome of vagus nerve stimulation for refractory partial epilepsy. Epilepsy Behav 4: 302-309.

30. Elliott RE, Morsi A, Tanweer O, Grobelny B, Geller E, et al. (2011) Efficacy of vagus nerve stimulation over time: Review of 65 consecutive patients with treatment-resistant epilepsy treated with VNS $>10$ years. Epilepsy Behav 20: 478-483.

31. De Ferrari GM, Crijns HJ, Borggrefe M, Milasinovic G, Smid J, et al. (2011) Chronic vagus nerve stimulation: A new and promising therapeutic approach for chronic heart failure. Eur Heart J 32: 847-855.

32. Premchand RK, Sharma K, Mittal S, Monteiro R, Dixit S, et al. (2014) Autonomic regulation therapy via left or right cervical vagus nerve stimulation in patients with chronic heart failure: Results of the ANTHEM-HF trial. J Card Fail 20: 808-816.

33. Zannad F, De Ferrari GM, Tuienenburg AE, Wright D, Brugada J, et al. (2015) Chronic vagal stimulation for the treatment of low ejection fraction heart failure: Results of the neural cardiac therapy for heart failure (NECTAR-HF) randomized controlled trial. Eur Heart J 36: 425-433.

34. Premchand RK, Sharma K, Mittal S, Monteiro R, Dixit S, et al. (2016) Extended follow-up of patients with heart failure receiving Autonomic Regulation Therapy in the ANTHEM-HF Study. J Card Fail 22: 639-642.

35. De Ferrari GM, Stolen C, Tuinenburg AE, Wright DJ, Brugada J, et al. (2017) Long-term vagal stimulation for heart failure: Eighteen month results from the neural cardiac therapy for heart failure (NECTAR-HF) trial. Int J Cardiol 244: 229-234.

36. Gold MR, Van Veldhuisen DJ, Hauptman PJ, Borggrefe M, Kubo SH, et al. (2016) Vagus nerve stimulation for the treatment of heart failure: The INOVATE-HF trial. J Am Coll Cardiol 68: 149-158.

37. LivaNova PLC. VNS Therapy® System Epilepsy Physician's Manual (US Version), January 2018.

38. BioControl Medical (BCM) Ltd. CardioFit ${ }^{\mathrm{TM}}$ Neurostimulator for Heart Failure Therapy Unified Physician Programmer Model 5300 Instructions for Use and TD. Document Number RU-53-001 Rev H dated 05 May 2008.

39. LivaNova PLC. VITARIA® System Physician's Manual (Non-US Version), November 2018.

40. DeGiorgio CM, Thompson J, Lewis P, Arrambide S, Nari- 
toku D, et al. (2001) Vagus nerve stimulation: Analysis of device parameters in 154 patients during the long-term XE5 study. Epilepsia 42: 1017-1020.

41. Heck C, Helmers SL, DeGiorgio CM (2002) Vagus nerve stimulation therapy, epilepsy, and device parameters: Scientific basis and recommendations for use. Neurology 59: S31-S37.

42. Yamamoto T (2015) Vagus nerve stimulation therapy: Indications, programing, and outcomes. Neurol Med Chir (Tokyo) 55: 407-415.

43. DiCarlo L, Libbus I, Amurthur B, KenKnight BH, Anand IS (2013) Autonomic regulation therapy for the improvement of left ventricular function and heart failure symptoms: The ANTHEM-HF study. J Card Fail 19: 655-660.

44. Hauptman PJ, Schwartz PJ, Gold MR, Borggrefe M, Van Veldhuisen DJ, et al. (2012) Rationale and study design of the increase of vagal tone in heart failure study: INOVATE-HF. Am Heart J 163: 954-962.

45. De Ferrari GM, Tuinenburg AE, Ruble S, Brugada J, Klein $\mathrm{H}$, et al. (2014) Rationale and study design of the neurocardiac therapy for heart failure study: NECTAR-HF. Eur J Heart Failure 16: 692-699.

46. Ardell JL, Nier H, Hammer M, Southerland EM, Ardell CL, et al. (2017) Defining the neural fulcrum for chronic vagus nerve stimulation: Implications for integrated cardiac control. J Physiol 595: 6887-6903.

47. Anholt TA, Ayal S, Goldberg JA (2011) Recruitment and blocking properties of the CardioFit stimulation lead. J Neural Eng 8: 034004.

48. Jewett DL (1964) Activity of single efferent fibres in the cervical vagus nerve of the dog, with special reference to possible cardio-inhibitory fibres. J Physiol 175: 321-357.

49. Writing Committee Members, Yancy CW, Jessup M, Bozkurt B, Butler J, et al. (2013) 2013 ACCF/AHA guideline for the management of heart failure: $A$ report of the American College of Cardiology Foundation/American Heart Association Task Force on practice guidelines. Circulation 128: e240-e327.

50. Libbus I, Nearing BD, Amurthur B, KenKnight BH, Verrier $\mathrm{RL}$ (2017) Quantitative evaluation of heartbeat interval time series using Poincaré analysis reveals distinct patterns of heart rate dynamics during cycles of vagus nerve stimulation in patients with heart failure. J Electrocardiol 50: 898903.

51. Anand IS, Konstam MA, Udelson JE, Butler J, Klein HU, et al. (2019) Vagus nerve stimulation for chronic heart failure: Differences in therapy delivery and clinical efficacy in ANTHEM-HF, INOVATE-HF, and NECTAR-HF. Journal of Cardiac Failure 25: S178.

52. Premchand RK, Sharma K, Mittal S, Monteiro R, Libbus I, et al. (2019) Long-term follow-up of reduced ejection fraction heart failure patients receiving autonomic regulation therapy in the ANTHEM-HF pilot study. Journal of the American College of Cardiology 73: 770.

53. Choi H, Sell RL, Lenert L, Muennig P, Goodman RR, et al. (2008) Epilepsy surgery for pharmacoresistant temporal lobe epilepsy: A decision analysis. JAMA 300: 2497-2505.

54. Cooke PM, Snider RS (1955) Some cerebellar influences on electrically-induced cerebral seizures. Epilepsia 4: 1928.

55. Sharan AD, Rezai AR (2009) Neurostimulation for Epilepsy. In: Krames ES, Peckham HP, Rezai AR, Neuromodula- tion. Elsevier, London, 617-666.

56. Fisher RS, Vickrey BG, Gibson P, Hermann B, Penovich $P$, et al. (2000) The impact of epilepsy from the patient's perspective I. Descriptions and subjective perceptions. Epilepsy Res 41: 39-51.

57. Labiner DM, Paradis PE, Manjunath R, Duh MS, Lafeuille $\mathrm{MH}$, et al. (2010) Generic antiepileptic drugs and associated medical resource utilization in the United States. Neurology 74: 1566-1574.

58. Helmers SL, Paradis PE, Manjunath R, Duh MS, Lafeuille $\mathrm{MH}$, et al. (2010) Economic burden associated with the use of generic antiepileptic drugs in the United States. Epilepsy Behav 18: 437-444.

59. Krahl SE, Clark KB (2012) Vagus nerve stimulation for epilepsy: A review of central mechanisms. Surg Neurol Int 3: S255-S259.

60. Schwartz PJ, Pagani M, Lombardi F, Malliani A, Brown AM (1973) A cardiocardiac sympathovagal reflex in the cat. Circ Res 32: 215-220.

61. Schwartz PJ, La Rovere MT, De Ferrari GM, Mann DL (2015) Autonomic modulation for the management of patients with chronic heart failure. Circ Heart Fail 8: 619-628.

62. Ardell JL, Andresen MC, Armour JA, Billman GE, Chen PS, et al. (2016) Translational neurocardiology: Preclinical models and cardioneural integrative aspects. J Physiol 594: 3877-3909.

63. McGuirt AS, Schmacht DC, Ardell JL (1997) Autonomic interactions for control of atrial rate are maintained after SA nodal parasympathectomy. Am J Physiol 272: $\mathrm{H} 2525-\mathrm{H} 2533$.

64. Standish A, Enquist LW, Schwaber JS (1994) Innvervation of the heart and its central medullary origin defined by viral tracing. Science 263: 222-234.

65. Kember G, Ardell JL, Shivkumar K, Armour JA (2017) Recurrent myocardial infarction: Mechanisms of free-floating adaptation and autonomic derangement in networked cardiac neural control. PLoS ONE 12: e0180194.

66. Ardell JL, Armour JA (2016) Neurocardiology: Structure-based function. Compr Physiol 6: 1635-1653.

67. Armour JA (2008) Potential clinical relevance of the 'little brain' on the mammalian heart. Exp Physiol 93: 165-176.

68. Libbus I, Mazar ST, Amurthur B, DiCarlo L, KenKnight B (2019) Quantitative assessment of transient heart rate dynamics in patients exposed to chronic autonomic regulation therapy provides evidence of durable neuromodulation resulting from cyclic stimulation of vagus nerve. Journal of the American College of Cardiology 73: 772.

69. Ben-Menachem E, Revesz D, Simon BJ, Silberstein S (2015) Surgically implanted and non-invasive vagus nerve stimulation: A review of efficacy, safety and tolerability. Eur J Neurol 22: 1260-1268.

70. Guiraud D, Andreu D, Bonnet S, Carrault G, Couderc P, et al. (2016) Vagus nerve stimulation: State of the art of stimulation and recording strategies to address autonomic function neuromodulation. J Neural Eng 13: 041002. 